gefallen, wenn die demoskopische Lage reelle Siegchancen versprochen hätte. Auf diese zentrale Weichenstellung hin passten alle anderen Akteure ihre Kalküle an, die schließlich das Wahlergebnis präformierten - ein Paradefall für die Pfadabhängigkeit politischer Prozesse. Ohne diesen speziellen Verlauf der Ereignisse wäre es sehr wahrscheinlich wieder zu einem ruinösen Wettlauf um das größte Bündnis und damit zu zwei sehr bunten Allianzen gekommen. Diese hätten wohl wieder fast alle abgegebenen Stimmen auf sich vereinigt, aber mit sich eine beträchtliche Anzahl von Kleinparteien ins Parlament gebracht. Bleibt das Wahlrecht bis zu den nächsten Wahlen unverändert, wird es wieder Anreize zu solchen großen Bündnissen geben. Ob die Politiker diesen Anreizen folgen oder sich wieder einer der Hauptakteure bereit findet, von sich aus auf den ruinösen Wettlauf zu verzichten, bleibt eine der vielen Unwägbarkeiten, von denen die italienische Politik auch nach den Parlamentswahlen 2008 gekennzeichnet ist.

Sollte sich der Trend von 2008 aber fortsetzen, könnte im italienischen Parteiensystem innerhalb von 20 Jahren eine zweite revolutionäre Umwälzung bevorstehen. Es besteht die Chance, dass von den Parteien, die 2006 zur Wahl standen, im Jahre 2013 erneut die meisten verschwunden sein werden. Das war, allerdings in einem kürzeren Zeitraum, schon zwischen 1992 und 1994 der Fall. Die einzige Partei, von der dies zum jetzigen Zeitpunkt kaum denkbar erscheint, ist die Lega Nord. Sie wird wohl nach Lage der Dinge auch 2013 als eigenständige Kraft antreten, in welcher Bündniskonstellation auch immer.

\title{
Erdrutsch als Oberflächenphänomen. Die Parlamentswahlen in Polen vom 21. Oktober 2007
}

\author{
Holger Münch
}

„Es geht ein Ruck durch Polen“, kommentierte der polnische Publizist Adam Krzemiński den Sieg der liberal-konservativen Bürgerplattform (PO) unter ihrem Spitzenkandidaten Donald Tusk über die bisherige Regierung unter Premierminister Jarostaw Kaczyński und seiner Partei Recht und Gerechtigkeit (PiS) in den Parlamentswahlen vom 21. Oktober 2007. ${ }^{1}$ Damit gab er der Erleichterung Ausdruck, die vor allem im westlichen Ausland und dabei insbesondere in Deutschland - über das Ende der international durch EU- und deutschlandfeindliche Ausfälle nicht sonderlich wohlgelittenen national-konservativen Koalition in Warschau artikuliert wurde. Wie bei der berühmten Ruckrede des ehemaligen Bundespräsidenten Roman Herzog gibt es jedoch auch beim polnischen Ruck wichtige Anhaltspunkte, dass die Stilisierung der Wahlen zu einer Zäsur in der Nachwendegeschichte nur in begrenztem Maße angebracht ist.

1 Adam Krzemiński, Nach der Polen-Wahl: Der Westen wird größer, in: Die Welt vom 29. Oktober 2007 (http://debatte.welt.de/kommentare/46236/nach+der+polenwahl+der+westen+wird+groess er, Abruf am 4. Mai 2008). 


\section{Die Ausgangslage vor den Wablen}

Die PiS-geführte Regierung kann als Opfer derselben Entwicklung bezeichnet werden, die sie 2005 an die Macht geführt hatte. Damals waren die Sozialdemokraten unter Premier Leszek Miller (vgl. Tabelle 1) in einem beispiellosen Sumpf aus Skandalen versunken. Im Zentrum stand eine Affäre um den Filmproduzenten Lew Rywin, der - angeblich im Auftrag der Links-Regierung - von dem Herausgeber der wichtigen Tageszeitung Gazeta Wyborcza als Gegenleistung für die Verabschiedung eines günstigen Mediengesetzes ein Schmiergeld in Millionenhöhe gefordert hatte. Im Zuge der zahlreichen Affären spaltete sich die Partei, während die Korruptionsdebatte und der angebliche Ausverkauf polnischer Interessen im Zuge des EU-Beitritts der Law and Order-Partei PiS und den Populisten der „Selbstverteidigung“, Samoobrona (SO), des Bauernführers Andrzej Lepper und der meist als ultrakatholisch bezeichneten Liga der Polnischen Familien (LPR) genug Treibstoff für ihre Kampagnen (sowohl für die Parlaments- als auch für die fast zeitgleich stattfindende Präsidentschaftswahl) lieferten. PiS gelang es dabei, diese Wahl in eine Entscheidung zwischen einem „sozialen und solidarischen“ Polen auf der einen Seite und einem liberalen Polen auf der anderen Seite umzudeuten. ${ }^{2}$ Dabei machte sich die Partei für eine Wiedereinführung der Todesstrafe, ein radikales Abtreibungsverbot und das Verbot homosexueller Beziehungen stark. Bei einer historisch und in Ostmitteleuropa einmalig niedrigen Wahlbeteiligung von wenig mehr als 40 Prozent wurde PiS im Herbst 2005 dann zur stärksten parlamentarischen Kraft, während die sozialdemokratische Linksallianz von 41 auf knapp elf Prozent abstürzte und damit sogar von Lepper und seiner SO überholt wurde. ${ }^{3}$

Das anschließende Scheitern der von allen Beobachtern erwarteten Koalitionsbildung von PiS und Bürgerplattform illustrierte einmal mehr die Fragmentierung und Zerrissenheit der politischen Szene und führte zunächst zu einer Minderheitsregierung unter dem unbekannten Kazimierz Marcinkiewicz. Dessen Nominierung war ein taktischer Schachzug Jarostaw Kaczyńskis, der sich zunächst nicht selbst zum Premier küren lassen wollte, um die Kandidatur seines Bruders Lech um das Präsidentenamt nicht zu gefährden. Es war nämlich zu befürchten, dass viele Polen bei der bevorstehenden Direktwahl des Staatsoberhauptes von einer - später dann doch noch realisierten - „Zwillingsherrschaft“ abgeschreckt werden könnten. Nachdem Lech im ersten Wahlgang lediglich den zweiten Platz belegen konnte, besiegte er seinen Rivalen Tusk von der Bürgerplattform in der Stichwahl. Eine wichtige Rolle spielte hier bereits die Methode der Diffamierung. So war im Wahlkampf verbreitet worden, Tusks Großvater hätte freiwillig in der deutschen Wehrmacht gedient, wäre mithin ein Vaterlandsverräter gewesen, obwohl dieser in Wahrheit zum Dienst gezwungen worden war.

Nach nur zehn Monaten im Amt wurde Marcinkiewicz im Juli 2006 von Jarostaw Kaczyński als Premier abgelöst. Von nun an standen die beiden konservativen Brüder gemeinsam an der Spitze des polnischen Staates, gestützt von einer starken Bewegung konservativer Intellektueller, auch einer sehr jungen Generation, und flankiert von der ultrakatholischen Rhetorik der LPR sowie dem diffus xenophoben Agro-Nationalismus der

2 Vgl. hierzu auch Aleks Szczerbiak, ,Social Poland' Defeats ,Liberal Poland'? The September-October 2005 Polish Parliamentary and Presidential Elections, in: Journal of Communist Studies and Transition Politics, 23. Jg. (2007), H. 2, S. $203-232$.

3 Alle Wahlergebnisse gemäß den Daten der polnischen Wahlkommission (www.pkw.gov.pl). 
Lepper-Partei. Diese Konstellation bildete die konservativste und zugleich europakritischste Regierung im Nachwende-Polen. Ihr Ziel war eine „IV. Republik“, die auf der Basis einer systematischen „Säuberung“ des polnischen Staates von der angeblich allgegenwärtigen Korruption und den angeblich herrschenden kommunistischen Seilschaften errichtet werden sollte. So wurde ein „Zentrales Anti-Korruptionsbüro“ gegründet, das direkt dem Ministerpräsidenten zugeordnet ist und als einer der offiziellen Nachrichtendienste Polens seine Aktivitäten unter weitgehendem Ausschluss der Öffentlichkeit vollzieht, so dass eine parlamentarische Kontrolle nur eingeschränkt möglich ist. Der durch Vetternwirtschaft, Korruption und politische Einflussnahme in Misskredit geratene militärische Geheimdienst (WSI) wurde aufgelöst. Wie bei den meisten Regierungen zuvor wurden zudem zentrale Ämter in der Verwaltung und in anderen wichtigen Institutionen, wie dem staatlichen Fernsehen, mit treuen Gefolgsleuten besetzt.

Innerhalb kurzer Zeit erzeugten die Kaczyńskis mit der systematischen Zentralisierung von Macht eine Atmosphäre des Misstrauens und der Angst in der Gesellschaft, wobei für sie der Zweck die Mittel heiligte und regelmäßig Gegner mit Intrigen aus dem Wege geräumt wurden. ${ }^{4}$ Eines der letzten Opfer wurde Koalitionspartner und Vize-Premier Lepper. Mitarbeiter des Anti-Korruptionsbüros wollten ihn verdeckt dazu verleiten, sich für die Umwandlung von Acker- in profitables Bauland bestechen zu lassen. Doch Lepper wurde anscheinend gewarnt und fiel nicht darauf herein. Trotzdem entließ ihn der Ministerpräsident aus der Regierung, die daraufhin zerbrach und suchte sein Heil bald darauf in der Flucht nach vorne. So stimmte die PiS (mit der PO) am 7. September 2007 für die Auflösung des Sejm, und Neuwahlen wurden ausgerufen.

Die Bilanz von rund zwei Jahren PiS-Regierung fiel nicht sonderlich positiv aus. Abgesehen von dem gescheiterten Kampf gegen die angeblich allgegenwärtige Korruption konnte die Regierung kaum Erfolge vorweisen. Trotz der ökonomisch günstigen Rahmenbedingungen mit einem Wirtschaftswachstum von über sechs Prozent 2006 und 2007 waren wichtige Reformen verschleppt worden. ${ }^{5}$ So blieb die dringend notwendige Gesundheitsreform aus. Zur Zeit der Regierungskrise im Sommer 2007 streikten wochenlang Ärzte und Krankenschwestern für höhere Gehälter und die Anhebung der Gesundheitsausgaben. Steuersenkungen und der Bau von Millionen neuer Wohnungen, im Wahlkampf 2005 versprochen, wurden nicht realisiert. Darüber hinaus hatten die Kaczyńskis mit ihrem konfrontativen Politikstil Polen international, vor allem in Europa, weitgehend isoliert und die deutsch-polnischen Beziehungen auf einen Tiefpunkt manövriert. Die „Kartoffelaffäre“ um eine Kaczcyński-Satire der Berliner „tageszeitung“ und die Aufrechnung der Stimmengewichtung im EU-Ministerrat gegen die polnischen Kriegstoten sind hierbei nur die schrillsten Beispiele.

Dennoch war der Ausgang der Wahl bis zur letzten Minute völlig offen. Die Umfrageergebnisse der verschiedenen Meinungsforschungsinstitute widersprachen sich so deutlich wie selten zuvor und riefen fast täglich eine neue Kräftekonstellation aus. Eine in der Gazeta Wyborcza veröffentlichte Umfrage sah noch am 11. Oktober 2007, also zehn Tage vor

4 Vgl. auch Gerhard Gnauck, Zwei Jahre PiS-Regierung - Versuch einer Bilanz, Polen-Analysen Nr. 19, 18. September 2007.

5 Vgl. Statistisches Hauptamt, Information on the socio-economic situation 2007 (http://www. stat.gov.pl/cps/rde/xbcr/gus/PUBL_Information_socio-economic_situation_country_2007.pdf, Abruf am 29. März 2008). 
den Wahlen, PiS mit 38 Prozent rund fünf Punkte vor der Bürgerplattform. Dies illustriert, dass die Stimmung in Polen bei weitem nicht so eindeutig war wie im europäischen Ausland. Vor allem bei ihrer Kernkompetenz, der Bekämpfung von Korruption und Kriminalität, wurde bislang keine andere polnische Regierung von den Bürgern so positiv bewertet;

\begin{tabular}{|c|c|c|}
\hline \multicolumn{3}{|c|}{ Tabelle 1: Regierungen in Polen seit 1989} \\
\hline Wahlperiode & Regierungskoalition & Ministerpräsident \\
\hline 1989 bis 1991 & $\begin{array}{l}\text { Große Koalition aus: Bürgerkomitee - ZSL - SD } \\
\text { - PVAP (Juli 1990: Abberufung der „Schlüsselmi- } \\
\text { nister“ der ehemaligen PVAP, die sich Anfang } 1990 \\
\text { aufgelöst hatte) } \\
\text { ab Januar 1991: } \\
\text { Koalition aus Personen und Gruppierungen der } \\
\text { Bürgerbewegung (unter anderem PC - KLD - } \\
\text { ZChN - SD - ROAD/UD) }\end{array}$ & $\begin{array}{l}\text { Tadeusz Mazowiecki (UD/UW) } \\
(12.09 .1989-12.01 .1991) \\
\text { Jan Krzysztof Bielecki (KLD) } \\
(12.01 .1991-23.12 .1991)\end{array}$ \\
\hline 1991 bis 1993 & $\begin{array}{l}\text { ZChN - PC - PChD - Solidarność der Privatbau- } \\
\text { ern (Unterstützung der Regierung durch PSL, ChD, } \\
\text { jedoch keine Regierungsbeteiligung) } \\
\text { ab 13. Juli 1992: } \\
\text { ZChN - PChD - UD - KLD - PL - SLCh - PPG }\end{array}$ & $\begin{array}{l}\text { Jan Olszewski (PC, später RdR, } \\
\text { dann ROP) (23.12.1991- } \\
04.06 .1992) \\
\text { Waldemar Pawlak (PSL) } \\
\text { (05.06.1992 - 08.07.1992) } \\
\text { Hanna Suchocka (UW) } \\
(11.07 .1992-17.10 .1993) \\
\end{array}$ \\
\hline 1993 bis 1997 & $\begin{array}{l}\text { SLD - PSL - UP - BBWR } \\
\text { ab 4. März 1995: } \\
\text { SLD - PSL }\end{array}$ & \begin{tabular}{|l|} 
Waldemar Pawlak (PSL) \\
$(18.10 .1993-01.03 .1995)$ \\
Józef Oleksy (SdRP/SLD) \\
$(01.03 .1995-25.01 .1996)$ \\
Wlodzimierz Cimoszewicz (SLD) \\
$(02.02 .1996-31.10 .1997)$ \\
\end{tabular} \\
\hline 1997 bis 2001 & $\begin{array}{l}\text { AWS (unter anderem Solidarność, PC, ZChN, } \\
\text { KPN, PL, PChD, BBWR, SKL) - UW } \\
\text { ab 1. Juni 2000: } \\
\text { AWS-Minderheitsregierung }\end{array}$ & $\begin{array}{l}\text { Jerzy Buzek (AWS) } \\
(31.10 .1997-19.10 .2001)\end{array}$ \\
\hline 2001 bis 2005 & $\begin{array}{l}\text { SLD/UP - PSL } \\
\text { ab 1. März 2004: } \\
\text { SLD/UP-Minderheitsregierung } \\
\text { (2. Mai 2004: Abspaltung der SdPL vom SLD) }\end{array}$ & $\begin{array}{l}\text { Leszek Miller } \text { (SLD) } \\
(19.10 .2001-02.05 .2004) \\
\text { Marek Belka (parteilos) } \\
(02.05 .2004-31.10 .2005)\end{array}$ \\
\hline 2005 bis 2007 & $\begin{array}{l}\text { PiS-Minderheitsregierung (Unterstützung durch SO } \\
\text { und LPR) } \\
\text { ab 5. Mai 2006: } \\
\text { Mehrheits-Koalition PiS - SO - LPR } \\
\text { ab 13. August 2007: } \\
\text { PiS-Minderheitsregierung }\end{array}$ & $\begin{array}{l}\text { Kazimierz Marcinkiewicz (PiS) } \\
(31.10 .2005-14.07 .2006) \\
\text { Jaroslaw Kaczyński (PiS) } \\
(14.07 .2006-16.11 .2007)\end{array}$ \\
\hline seit 2007 & $\mathrm{PO}-\mathrm{PSL}$ & $\begin{array}{l}\text { Donald Tusk (PO) } \\
\text { (seit 16.11.2007) }\end{array}$ \\
\hline \multicolumn{3}{|c|}{$\begin{array}{l}\text { ZSL: Bauernpartei, SD: Demokratische Partei, PVAP: Vereinigte Arbeiterpartei, PChD: Partei der } \\
\text { Christdemokraten, ChD: Christdemokratie, PL: Bauernverständigung, SLCh: Ex-Solidarność der Privat- } \\
\text { bauern (später SKL) - PPG: Polnisches Wirtschaftsprogramm, BBWR: Unparteiischer Block zur Unter- } \\
\text { stützung der Reformen, für weitere Parteinamen siehe Legende von Tabelle } 2 \text {. } \\
\text { Quelle: Holger Münch, Leitbilder und Grundverständnisse der polnischen Europapolitik, Wiesbaden } \\
\text { 2007, S. 68, mit Aktualisierung. }\end{array}$} \\
\hline
\end{tabular}


aber auch die - während der Koalition von Lepper verantwortete - Landwirtschaftspolitik schnitt recht gut ab. ${ }^{6}$ Die Kaczyńskis hatten sich in den vorangegangenen zwei Jahren den Ruf erworben, zwar grob, aber unbestechlich zu sein und kompromisslos zu ihren Überzeugungen zu stehen. Und nicht nur von der potentiellen PiS-Wählerschaft wurde zudem die unnachgiebige Haltung gegenüber den europäischen Partnern, und allen voran Deutschland, durchaus positiv aufgenommen. Zudem hätte der Premier kaum die Auflösung des Parlaments initiiert, wenn er nicht von seiner Wiederwahl überzeugt gewesen wäre.

\section{Der Wahlkampf}

Der Wahlkampf war einer der kürzesten seit 1989. Zwischen der Auflösung des Sejm und dem Wahltermin am 21. Oktober 2007 lagen nur sechs Wochen. Angesichts dieser kurzen Zeitspanne, der starken Polarisierung der politischen Kräfte und der offensichtlichen Unentschiedenheit vieler polnischer Wähler wurde die Auseinandersetzung mit beispielloser Härte geführt, so dass dieser Wahlkampf auch als einer der aggressivsten seit 1989 gelten kann. Da die Meinungsforscher den beiden kleineren Partnern der alten Koalition einhellig ein Scheitern voraussagten, hatten die bäuerliche SO und die katholische PR nichts mehr zu verlieren. Lepper war ohnehin durch die Korruptionsvorwürfe geschwächt. Nachdem er zwei Mal als Minister entlassen worden und im Anschluss an seinen letzten Hinauswurf trotzdem für ein Verbleiben seiner Partei in der Koalition eingetreten war, hatte er einen weiteren Teil seiner Glaubwürdigkeit verspielt. So belegte er im Wahlmonat den zweiten Platz auf der Liste der Politiker, denen die Polen am stärksten misstrauten. ${ }^{7}$

Auf Platz eins fand sich der Chef der LPR, Roman Giertych. Er versuchte im Wahlkampf, seine Partei als singuläre nichtkorrupte politische Kraft Polens darzustellen: Nur sie sei nicht an der Aushandlung des „faulen Kompromisses III. Republik“ am Runden Tisch beteiligt gewesen, bei dem PO, PiS und die Sozialdemokraten (beziehungsweise Kommunisten) die Macht im Lande unter sich aufgeteilt hätten. Die LPR sei beim Eintritt in die Koalition auf die leeren Versprechungen der Kaczyńskis hereingefallen, die nicht weniger korrupt seien als die Liberalen und Kommunisten, mit denen sie an einem Strang zögen. ${ }^{8}$ Neben dieser LPR-typischen Verschwörungsargumentation gab es Verhandlungen, ein Bündnis mit Lepper zu schmieden, um die Erfolgschancen zu erhöhen. Für ein solches Bündnis, das den Namen LiS (Liga i Samoobrona) tragen sollte, hätte jedoch die höhere Sperrklausel von acht Prozent gegolten, die angesichts der Umfragewerte unüberwindlich scheinen musste. Letztlich holte die LPR die „Rechte der Republik“ (Prawica Rzeczspospolita) und die Union für Realpolitik (UPR) ins Boot, die rechtsradikalen Gruppierungen nahesteht und antisemitische Tendenzen aufweist. ${ }^{9}$ Dabei wurde kein Wahlbündnis gegründet, um von der Fünf-Prozent-Sperrklausel für eine einfache Parteienliste unter dem Schild der Liga zu profitieren.

6 Vgl. CBOS, Polish Public Opinion, September 2007 (http://www.cbos.pl/PL/Opinia/2007/09 _2007.pdf).

7 CBOS, Komunikat z Badań, BS 159/2007, Warschau, Oktober 2007 (http://www.cbos.pl).

8 Vgl. die Rede von Roman Giertych anlässlich der parlamentarischen Debatte zur Auflösung des Sejm bei dessen 47. Sitzung am 7. September 2007 (http://www.sejm.gov.pl).

9 Vgl. Holger Münch, Leitbilder und Grundverständnisse der polnischen Europapolitik, Wiesbaden 2007, S. 79. 
So wie die Kampagnen von SO und LPR folgte auch der Wahlkampf von PiS der Logik des siegreichen Wahljahres 2005. Bewusst wurde dabei die Wahl zu einem Plebiszit über die bisherige Regierungszeit aufgebaut. Es gehe darum, so die Argumentation, den Kampf gegen die Korruption und die alten Seilschaften fortzusetzen, in dem man zwar bereits wichtige Siege habe erringen können, der aber längst noch nicht beendet sei. Ein Regierungswechsel würde unwiderruflich die Wiederherstellung des alten „Filzes“ bewirken. Dabei entdeckte der Chef der PiS neben den ominösen kommunistischen Seilschaften auch die so genannten Privilegierten als nationale Bedrohung, die er später gar als „Oligarchen“ bezeichnete, was mancher Beobachter als weiteres Zeichen einer „Putinisierung“ der polnischen Politik wertete. ${ }^{10}$

Letztlich wurde die Auseinandersetzung insgesamt weniger über konkrete politische Differenzen zwischen den Parteien ausgetragen, sondern eher über solche allgemeinen Drohkulissen und Negativkampagnen, die häufig allein auf die persönliche Diffamierung politischer Gegner abzielten (wobei PiS und PO die Hauptkontrahenten darstellten). ${ }^{11}$ Auch die anti-deutsche Karte wurde wieder gespielt: So warf Premier Kaczyński der Bürgerplattform eine Abhängigkeit von den Deutschen und die Akzeptanz der deutschen Dominanz in Europa vor.

Unterstützung bei ihrer Kampagne erhielt die Regierung vom radikal-klerikalen Sender Radio Maryja, der ein wichtiger Meinungsbildner im ländlichen Polen ist (und regelmäßig durch antisemitische und homophobe Äußerungen auffällt). Zudem mischte sich der Präsident mehrfach aktiv auf Seiten der PiS in den Wahlkampf ein, und das staatliche Fernsehen billigte den Vertretern der Regierung unverhältnismäßig viel Sendezeit zu, was später die Wahlbeobachter der OSZE auch in ihrem Abschlussbericht monierten. ${ }^{12}$

Ein weiteres zentrales Wahlkampfinstrument in den Händen der Kaczyńskis stellte das bereits erwähnte Anti-Korruptionsbüro (CBA) dar, das mit einem fingierten Bestechungsversuch schon beim Kampf gegen Koalitionspartner Lepper zum Einsatz gekommen war. So kündigte Zbigniew Ziobro, Justizminister und Generalstaatsanwalt in Personalunion, bereits vor der Auflösung des Parlaments an, „Beweise“ über angebliche kriminelle Aktivitäten der Oppositionsführer vorlegen zu wollen, einschließlich Tusk von der Bürgerplattform, Wojciech Olejniczak von der Sozialdemokratie (LiD) sowie Giertych und Lepper. Die Bürgerplattform erstattete daraufhin Anzeige gegen den Justizminister wegen Überschreitung seiner Befugnisse, und der polnische Bürgerrechtsbeauftragte forderte aufgrund des offensichtlichen Missbrauchs staatlicher Organe für den Wahlkampf die Einladung internationaler Wahlbeobachter.

Hinter dem Duell der beiden großen Parteien traten die Kampagnen der kleineren Gruppierungen zurück. Hier sind zunächst die Linksdemokraten (LiD) als Zusammenschluss aus vier linken beziehungsweise liberalen Gruppierungen zu nennen (und zwar aus den ehemals regierenden Sozialdemokraten (SLD) und ihrer Abspaltung SDPL sowie der

10 Vgl. hierzu auch: Gerhard Gnauck, a.a.O., S. 5.

11 Bezeichnend erscheint hier ein Wahlspot der PiS, in dem ein Unternehmer einem Politiker einen Koffer voller Dollar-Noten überreicht und danach seinen Partner anruft, um ihm mitzuteilen, dass man den Regierungsauftrag erhalten hätte und nun noch die Opposition bestechen müsse.

12 OSCE, Republic of Poland Pre-Term Parlamentary Elections 21 October 2007. OSCE/ODIHR Election Assessment Final Mission Report, Warsaw 20 March 2008, S. 12, S. 15 ff. 
sozialistischen Arbeitsunion und der Demokratischen Partei (PD), der Nachfolgerin der zu einem wichtigen Teil in der PO aufgegangenen Freiheitsunion). Ihr Wahlprogramm unter dem Titel „Neue Politik, neuer Staat“ transportierte sozialdemokratische Inhalte und präsentierte das Bündnis als Wahrerin rechtsstaatlicher Standards, die bei einem Sieg durch eine eigene „Charta" geschützt werden sollten. Bei der Wirtschaftspolitik wurde vor allem der Stellenwert der Ausbildung betont, die als zentraler Faktor eines hohen Wirtschaftswachstums ausgemacht wurde, das heißt, hier bleibt der Text erwartungsgemäß unverbindlich angesichts der unterschiedlichen Auffassungen der Bündnismitglieder. ${ }^{13}$

Als Galionsfigur der LiD zog Ex-Präsident Aleksander Kwaśniewski in den Wahlkampf. Im Fernsehduell mit Tusk erzielte er beinahe ein Remis, jedoch litt seine Glaubwürdigkeit beziehungsweise die der Kampagne unter streitbaren Auftritten, bei denen er einen angetrunkenen Eindruck machte (was besonders ungünstig wirkte, da er bereits in seiner Amtszeit diverse Male offensichtlich unter Alkoholeinfluss öffentlich aufgetreten war). Zudem war lange nicht klar, welche Position das ehemalige Staatsoberhaupt bei einer Regierungsbeteiligung der LiD einnehmen würde. Zunächst hieß es, er wolle sich nach der Wahl aus der Politik zurückziehen, nach einer Weile legte er sich aber darauf fest, im Bedarfsfalle doch als möglicher Ministerpräsident zur Verfügung zu stehen.

Die Kampagne der traditionellen Bauernpartei PSL orientierte sich am ehesten an Sachthemen. In ihrem Wahlprogramm „Gemeinsam gestalten wir eine bessere Zukunft“ dominierten Wirtschaftsfragen, wobei eine prononciert interventionistische Politik und ein starker Sozialstaat befürwortet wurden. ${ }^{14}$ Gleichzeitig suchte sich die PSL als moderne Partei zu präsentieren und strebte die Möglichkeit zur Registrierung von Firmen über das Internet sowie die Schaffung von Telearbeitsplätzen an, um den Menschen eine Arbeit „nahe am Zuhause“ zu ermöglichen. Auch die Erhöhung des Anteils an erneuerbaren Energien durch eine Umstellung des landwirtschaftlichen Anbaus schrieb sie sich auf die Fahnen. Gleichzeitig stimmte der amtierende Vorsitzende Waldemar Pawlak in die allgemeine KaczyńskiKritik ein und kritisierte sowohl die konfrontative „Wahlpropaganda“ von PiS als auch das während ihrer Regierungszeit geschaffene politische System in Polen, das einer „Demokratura" zwischen der II. Republik (der Zwischenkriegszeit) und der kommunistischen Zeit gleiche. ${ }^{15}$

In der letzten Woche vor den Wahlen erhitzte sich die Debatte nochmals deutlich. Die politische Mission des Anti-Korruptionsbüros trat deutlich zutage, als deren Chef Mariusz Kaminski auf einer Pressekonferenz über Korruptionsermittlungen gegen eine PO-Abgeordnete berichtete und die Bevölkerung explizit dazu aufforderte, diese Informationen bei ihrer Wahlentscheidung zu berücksichtigen. Die Programmverantwortlichen des staatlichen Senders TVP1 passten den Sendeablauf eigens auf dieses Ereignis hin an. Letztlich erwies sich jedoch die Instrumentalisierung von Medien und Justiz für politische Zwecke als Bumerang für die Regierung. So schlug eine Abgeordnete von Leppers SO die Regierungspartei mit ihren eigenen Mitteln, als sie heimlich einen Versuch von Kaczyńskis Kanzleileiter

13 Vgl. 100 Konkretów - Program wyborczy LiD, http://www.sld.org.pl/ index.php?view= 1\&art_ id=13567\&pid=18\&ret_id=48\&rsid=0, Abruf am 25. Mai 2008.

14 Vgl. Program Wyborczy PSL, in: Wirtualna Polska (http://wybory2007.wp.pl/wid,9242706, wiadomosc.html, Abruf am 25. Mai 2008).

15 Zitiert nach: PSL: Mamy demokraturę, nie demokrację, in: Gazeta Wyborcza vom 10. Oktober 2007 (http://miasta.gazeta.pl/krakow/1,35824,4566918.html, Abruf am 27. Mai 2008). 
filmen ließ, der sie mit Geld und der Aussicht auf einen Regierungsposten zur Überläuferin machen wollte. Nach der Veröffentlichung der Aufnahmen sagte der Vorsitzende der PSL postwendend geplante Sondierungsgespräche über eine Koalitionsbildung mit der PiS ab. Die Kaczyński-Partei hatte sich damit praktisch selbst des letzten potentiellen Partners für die Zeit nach den Wahlen beraubt.

Weitaus wichtiger war jedoch das TV-Duell zwischen dem Noch-Premier und seinem Konkurrenten Tusk. Dieser überraschte mit Angriffslust und Schlagfertigkeit und wurde nach Umfragen eindeutiger Sieger ${ }^{16}$, was der PO neuerlichen Schub in dem wochenlangen Kopf-an-Kopf-Rennen mit dem politischen Gegner gab und die Anhänger mobilisierte. Diesen beginnenden Stimmungswandel verstärkten prominente Autoritäten des Landes wie der ehemalige Außenminister Wtadystaw Bartoszewski, der offen zur Wahl der PO aufrief. Hinzu kamen interne Querelen bei PiS, wo in Ungnade gefallene Leitfiguren wie der entlassene und des Geheimnisverrats bezichtigte Innenminister Janusz Kaczmarek in der Öffentlichkeit schmutzige Parteiwäsche wuschen und die Kaczyńskis öffentlich des Amtsmissbrauchs zu politischen Zwecken beschuldigten. ${ }^{17}$ Eine weitere Rolle spielten gewichtige Parteiaustritte wie der des ehemaligen PiS-Premiers Marcinkiewicz, der aus „moralischen Gründen“"sein Parteibuch zurückgab.

\section{Das Wahlergebnis}

30,5 Millionen Wahlberechtigte ab 18 Jahren waren aufgerufen, in den sechsten vollständig freien Wahlen seit der demokratischen Wende 1989 am 21. Oktober 2007 ein neues Parlament zu wählen. Dieses besteht aus zwei Kammern, dem Sejm, dem zentralen legislativen Organ mit 460 Abgeordneten, und dem hundertköpfigen Senat, der Änderungen zu Gesetzesvorlagen des Sejm vorschlagen kann, bevor diese zur Unterzeichnung an den Präsidenten weitergeleitet werden. Die Mitglieder des Sejms werden nach einem Verhältnissystem in 41 Mehrpersonenwahlkreisen gewählt, wobei pro Wahlkreis sieben bis 19 Mandate vergeben werden. ${ }^{18}$ Es gilt eine Sperrklausel von fünf Prozent für Parteien und acht Prozent für Wahlbündnisse. Die deutsche Minderheit ist von dieser Regelung ausgenommen. Der Senat wird per Mehrheitswahl besetzt, wobei 40 Wahlbezirke mit jeweils zwei bis vier Senatoren vorgesehen sind. Die Festlegung der Wahlbezirke auf 41 (Sejm) beziehungsweise 40 (Senat) ist ebenfalls Folge des 2001 modifizierten Wahlgesetzes, das die bislang neben den Listen für die Einzelwahlkreise bestehende einheitliche Landesliste abschaffte und die

16 Gorąca debata Kaczyński- Tusk - Kto wygrał?, in: Gazeta Wyborcza Online vom 12. Oktober 2007 (http://wiadomosci.gazeta.pl/Wiadomosci/1,80708,4574515.html?as=4\&ias=4\&startsz=x); Sondaże: Miażdżące zwycięstwo Tuska, in: ebenda (zuletzt aktualisiert am 15. Oktober 2007, http://wiadomosci.gazeta.pl/Wiadomosci/1,80708,4574526.html), vgl. auch Stephan Raabe, Wende im polnischen Wahlkampf, KAS-Länderbericht vom 15. Oktober 2007 (http://www.kas. de/wf/doc/kas_12101-544-1-30.pdf).

17 Thomas Urban, Kaczynski am Pranger, in: Süddeutsche Zeitung vom 24. August 2007.

18 Die Berechnung erfolgte bis zur Änderung des Wahlgesetzes im April 2001 nach d'Hondt; zwischendurch wurde ein modifiziertes Saint-Laguë-Verfahren angewendet, womit die Überrepräsentation der großen Parteien verringert werden sollte, und im Juli 2002 kehrte man auf Betreiben des regierenden Linksbündnisses wieder zu d'Hondt zurück. 
Wahlgeographie im Zuge der Gebietsreform von 1999 (Aufteilung Polens in 16 Wojewodschaften) durch eine Verkleinerung der bisherigen 52 Wahlkreisen neu definierte. ${ }^{19}$

Eine Besonderheit des passiven Wahlrechts, das ab dem 21. Lebensjahr ausgeübt werden kann, besteht in der durch das Lustrationsgesetz festgesetzten Pflicht für alle vor dem 1. August 1972 Geborenen, Auskunft über eine mögliche Kollaboration mit den ehemaligen kommunistischen Sicherheitsdiensten erteilen zu müssen. Dabei hat nicht die Tatsache einer solchen Zusammenarbeit den Ausschluss vom passiven Wahlrecht zur Folge, sondern nur die Erteilung falscher Angaben. Sollte ein Gerichtsurteil eine entsprechende Lüge im Lustrationsverfahren bestätigen, so hätte dies eine "Wahlsperre“ des so Überführten für zehn Jahre zur Folge. ${ }^{20}$

Insgesamt hatten die Bürger bei der Besetzung des Sejm landesweit die Wahl zwischen sechs Parteilisten: Bürgerplattform (PO), Recht und Gerechtigkeit (PiS), Liga der Polnischen Familien (LPR), Selbstverteidigung (SO), Polnische Volkspartei (PSL) und Polnische Arbeitspartei (PPP). Hinzu kamen das Wahlbündnis der Linksallianz und einige Listen, deren Mitglieder nur in ausgewählten Bezirken wählbar waren, wie die Frauenpartei und die Deutsche Minderheit. Für den Senat traten Kandidaten auf insgesamt 29 Listen an, dabei lediglich sieben Gruppierungen in mehr als zehn Prozent der Wahlkreise.

\subsection{Wahlbeteiligung, Sitzverteilung und Wählerwanderung}

Die erste große Überraschung des Wahlabends war die für Polen ungewöhnlich hohe Wahlbeteiligung von 53,9 Prozent. Kein Wahlgang seit 1989 hat mehr Bürger an die Urnen gelockt. Insgesamt waren es 16,5 Millionen; 2005 stellte mit lediglich 12,3 Millionen (40,6 Prozent) einen Tiefpunkt der Partizipation dar. Bei der ersten Europawahl, an der Polen teilnahm, hatten sogar nur rund 38 Prozent ihre Stimme abgegeben. Besonders hoch war die Mobilisierung mit über 60 Prozent in Masowien, der Region um Warschau. In einigen Stadtteilen der Hauptstadt lag die Beteiligung sogar bei rund 90 Prozent, so dass vielerorts die Stimmzettel ausgingen, was Verzögerungen im Wahlablauf zur Folge hatte und zu zahlreichen Beschwerden bei der Wahlkommission führte. ${ }^{21}$ Auch die im Ausland lebenden Polen beteiligten sich mit fast 80 Prozent ungewöhnlich stark. Da ihre Stimmen jedoch nur für den Wahlkreis Warschau gezählt wurden, waren sie nicht wahlentscheidend.

Klarer Wahlsieger wurde die Bürgerplattform unter Spitzenkandidat Tusk. Mit 41,5 Prozent verdoppelte sie gegenüber 2005 ihren Stimmenanteil, im Vergleich zu der Parlamentswahl von 2001 vervierfachte sie ihn sogar (vgl. Tabelle 2$).{ }^{22}$ Dies stellt zudem das beste

19 Die ebenfalls neu geregelte Parteienfinanzierung sieht eine staatliche Förderung von Parteien vor, die mindestens drei Prozent Wählerstimmen erhalten, wobei sich der Förderbetrag nach der Zahl der abgegebenen Stimmen richtet. Spenden von Unternehmen sind den Parteien ebenso verboten wie Einnahmen durch gewerbliche Tätigkeiten oder die Vermietung von Immobilien. Vgl. hierzu die Wahlordnung des Sejm und des Senats vom 12. April 2001 (http://www.sejm.gov.pl/prawo/ nowaord/kon11.htm).

20 Dieser lange Zeitrahmen wurde zwischenzeitlich vom Verfassungstribunal moniert, das in seiner Entscheidung vom 11. Mai 2007 bereits eine Änderung verlangt hat.

21 Vgl. Janusz A. Majcherek, Die polnischen Parlamentswahlen. Eine Analyse des Ergebnisses, PolenAnalysen Nr. 23, 20. November 2007, S. 2 f.

22 Vgl. auch Andrea Gawrich, Die jüngsten Parlamentswahlen in Polen, Ungarn und Tschechien, in: ZParl, 34. Jg. (2003), H. 2, S. $270-284$. 
Tabelle 2: Ergebnisse der Sejmwablen in Polen 1997 bis 2007

\begin{tabular}{|c|c|c|c|c|c|c|c|c|}
\hline & \multicolumn{2}{|c|}{1997} & \multicolumn{2}{|r|}{2001} & \multicolumn{2}{|r|}{2005} & \multicolumn{2}{|r|}{2007} \\
\hline & Sitze & $\begin{array}{l}\text { Stimmen } \\
\text { in Prozent }\end{array}$ & Sitze & $\begin{array}{c}\text { Stimmen in } \\
\text { Prozent }\end{array}$ & Sitze & $\begin{array}{l}\text { Stimmen in } \\
\text { Prozent }\end{array}$ & Sitze & $\begin{array}{l}\text { Stimmen in } \\
\text { Prozent }\end{array}$ \\
\hline $\mathrm{PO}$ & - & - & 65 & 12,68 & 133 & 24,14 & 209 & 41,51 \\
\hline PiS & - & - & 44 & 9,50 & 155 & 26,99 & 166 & 32,11 \\
\hline PSL & 27 & 7,31 & 42 & 8,98 & 25 & 6,96 & 31 & 8,91 \\
\hline $\mathrm{LiD}$ & - & - & - & - & & & 53 & 13,15 \\
\hline $\begin{array}{l}\text { SLD (ab } 2001 \\
\text { SLD / UP) }\end{array}$ & 164 & 27,13 & 216 & 41,04 & 55 & 11,31 & & \\
\hline UP & - & 4,74 & - & - & - & - & - & - \\
\hline $\mathrm{SO}$ & - & 0,08 & 53 & 10,20 & 55 & 11,41 & - & 1,53 \\
\hline LPR & - & - & 38 & 7,87 & 34 & 7,97 & - & 1,3 \\
\hline AWS / AWSP & 201 & 33,83 & - & 5,60 & - & - & - & - \\
\hline $\begin{array}{l}\text { UW (ab 2005: } \\
\text { PD) }\end{array}$ & 60 & 13,37 & - & 3,10 & - & 2,5 & - & - \\
\hline ROP & 6 & 5,56 & - & - & - & - & - & - \\
\hline $\begin{array}{l}\text { Deutsche } \\
\text { Minderheit }\end{array}$ & 2 & 0,61 & 2 & 0,36 & 2 & 0,29 & 1 & 0,20 \\
\hline
\end{tabular}

Abkürzungen: AWS: Akcja Wyborcza Solidarność (Wahlaktion Solidarität. Erst 1996 gegründet, zerfiel sie am Ende der auf die Wahlen von 1997 folgende Wahlperiode und trat 2001 nicht mehr an. Aus ihr gingen die PiS sowie der konservative Flügel der PO hervor (deren liberaler Flügel schwerpunktmäßig aus ehemaligen UW-Mitgliedern besteht). Der Kern startete als AWS Prawicy 2001, scheiterte jedoch an der für Wahlbündnisse geltenden Acht-Prozent-Hürde.); LiD: Lewica i Demokraci (Wahlbündnis, bestehen aus SLD, SDPL, UP und PD (welche aus der UW hervorgegangen ist); LPR: Liga Polskich Rodziń (Liga der Polnischen Familien; trat wie die SO erstmals 2001 zu Parlamentswahlen an); PiS: Prawo i Sprawiedliwość (Recht und Gerechtigkeit; ging 2001 aus der zerfallenden AWS hervor und trat 2001 erstmals eigenständig bei Wahlen an); PO: Platforma Obywatelska (Bürgerplattform; trat ebenfalls als Auffangbecken für Kräfte aus AWS und der Freiheitsunion (UW) erstmalig 2001 an); PSL: Polskie Stronnictwo Ludowe (Polnische Bauernpartei); ROP: Ruch Odbudowy Polski (Bewegung zum Wiederaufbau Polens; startete 2001 von der Liste der LPR aus, war ab 2005 nicht mehr im Sejm vertreten); SDPL: Socjaldemokracja Polska (Polnische Sozialdemokratie; spaltete sich im Zuge des Niedergangs des SLD ab); SLD: Sojusz Lewicy Demokratycznej (Bündnis der Demokratischen Linken); SO: Samoobrona (Selbstverteidigung); UP: Unia Pracy (Arbeitsunion; trat ab 2001 von der Liste des SLD an, die sich SLD/UP nannte.)

Quelle: Eigene Zusammenstellung nach Daten der Nationalen Polnischen Wahlkommission, Warschau (http://www.pkw.gov.pl).

Ergebnis eines polnischen Wahlsiegers seit 1989 dar. Im Senat ist die Situation ebenso eindeutig. Hier konnte Tusks Partei 60 der 100 Sitze erringen (im Vergleich zu 34 im Jahre 2005). Die PiS kam lediglich auf 39 und verlor somit zehn Senatoren. ${ }^{23}$ Im Sejm konnte Kaczyńskis Partei jedoch weiter zulegen. Mit insgesamt 32,1 Prozent vergrößerte sie ihren Stimmenanteil um rund 5 Prozentpunkte, was im Vergleich zu 2001 eine Verdreifachung bedeutet. Das Linksbündnis unter der Führung Kwaśniewskis und des SLD blieb mit 13,2

23 Den verbleibenden 100. Sitz errang der ehemalige SLD-Außenminister Wtodzimierz Cimoszewicz, der als unabhängiger Kandidat angetreten war („Wahlkomitee Cimoszewicz in den Senat"). 
Prozent deutlich hinter den angepeilten 20 Prozent zurück, verbesserte sich jedoch im Vergleich zu den letzten Wahlen um zwei Prozentpunkte. Ihr Gewicht macht jedoch nur noch rund ein Drittel des 2001 errungenen Stimmenanteils aus. Diese gravierenden Verschiebungen bei den drei größten Parteien illustrieren eindrucksvoll die bedeutenden Wandlungsprozesse, denen das Parteiensystem in Polen unterworfen ist.

Ein weiteres Indiz für die hohe Fluktuation in der politischen Landschaft ist der Absturz der ehemaligen PiS-Koalitionäre Samoobrona und Liga der Polnischen Familien. Ersterer wurde mit gerade einmal 1,5 Prozent aus dem Sejm gefegt, die LPR schnitt mit 1,3 Prozent noch schlechter ab. Auch im Senat sind diese Gruppierungen nicht mehr vertreten. Viele Kommentatoren rechnen das Verdienst für diesen vernichtenden Schlag gegen die populistisch-nationalistischen Kräfte dem scheidenden Premier Kaczyński zu. Tatsächlich hatte dieser bereits im Vorfeld des Referendums zum EU-Beitritt 2004 angekündigt, diese durch Erschließung ihrer Wählerschaft überflüssig machen zu wollen. Durch die Einbindung von $\mathrm{SO}$ und LPR in seine Regierung ist ihm so gelungen, woran andere etablierte Parteien Europas gescheitert sind, nämlich extreme Gruppierungen durch Entzauberung in die Bedeutungslosigkeit zu verbannen.

Dabei ging auch das Kalkül auf, breite Wählerschaften aus diesem rechten Lager zu übernehmen. So wechselten rund 400.000 von der Liga zu PiS, rund 360.000 konnte Kaczyński Leppers Selbstverteidigung abspenstig machen. Zudem kam der PiS die hohe Wahlbeteiligung zugute: Sie gewann rund 1,8 Millionen neue Stimmen hinzu. All dies konnte jedoch die Niederlage nicht verhindern, denn noch stärker als die PiS profitierte die PO von der hohen Partizipation. 2,5 Millionen neue Wähler konnte sie hinzugewinnen, und Tusk verlor zwar über 100.000 Stimmen an Kaczyński, jedoch erhielt er im Gegenzug von der PiS rund eine halbe Million hinzu. Und auch vom Niedergang Leppers profitierte der Wahlsieger: Knapp 180.000 wechselten zur PO, von der LPR kamen mit circa 80.000 deutlich weniger. Auch am anderen Ende der politischen Skala ging Tusk erfolgreich auf Stimmenfang und holte fast eine Million ehemaliger Linkswähler in das Lager seiner Partei. Die LiD konnte diesen Stimmenverlust durch die hohe Mobilisierung ausgleichen, der erhoffte Zugewinn blieb jedoch aus. Die PSL wiederum legte als kleine Wahlsiegerin um rund 600.000 Stimmen zu und verlor nur knapp 100.000 an PO und PiS.

\subsection{Wahlverhalten nach Bevölkerungsgruppen und Regionen}

Die Wählerschaften der Parteien lassen sich relativ gut nach Alter, Bildungsgrad und örtlicher Herkunft unterscheiden. Regional zeigt sich eine klare Ost-West-Teilung. So war die PiS vor allem in den östlichen Gebieten erfolgreich. In Łódz, Masowien und in Małopolska siegte sie in den ländlichen Regionen, in den Städten gewann die Plattform. PiS hat somit den stärksten Rückhalt in den am wenigsten entwickelten und urbanisierten Regionen, die durch eine schwache Infrastruktur und eine geringe Dichte an akademischen Einrichtungen geprägt sind. Entsprechend ist auch der Anteil an ausländischen Direktinvestitionen in diesen Gebieten am niedrigsten. ${ }^{24}$ In den höher entwickelten Gebieten im Westen sowie in

24 Vgl. Bundesagentur für Außenwirtschaft (bfai), Polen wirbt für Investitionen in östliche Landesteile (Artikel aus der Datenbank Länder und Märkte), 25. Mai 2007 (www.bfai.de, Abruf am 25. Mai 2008). 
den Großstädten (Warschau, Posen, Stettin, Breslau, Krakau etc.) dominierte klar die PO. So wählten über 50 Prozent in den Städten mit mehr als 200.000 Einwohnern PO und unter 30 Prozent PiS. In Warschau trat Tusk direkt gegen Kazcyński an und triumphierte klar mit 46,6 gegen 23,9 Prozent. Er behielt die Nase auch in kleineren Städten vorn, selbst in solchen mit weniger als 50.000 Einwohnern. Nur auf dem Land hatte die PiS mit knapp 40 Prozent einen Vorsprung von rund zehn Punkten vor der PO. Ähnlich gestaltet sich das Bild bei der PSL, die als klassische Bauernpartei ebenfalls auf dem Land mit rund zwölf Prozent Zustimmung um ein Mehrfaches stärker ist als in den Großstädten, in denen sie mit drei Prozent praktisch keine Rolle spielt. Die Linksallianz ähnelt hier als Partei der Städte eher der PO, wobei der LiD-Schwerpunkt vor allem in den Städten zwischen 50.000 und 200.000 Einwohnern zu finden ist. ${ }^{25}$

Diese regionale Verteilung legt den Schluss nahe, dass die Bürgerplattform und die Linksallianz ihre Gefolgschaft vor allem in den gut ausgebildeten urbanen Milieus finden, während die Anhänger Kaczyńskis und der PSL vor allem unter den weniger Gebildeten zu finden sind. Tatsächlich schnitt die PO am besten bei Wählern mit höherer Bildung ab. Mit über 50 Prozent unterstützte in dieser Gruppe ungefähr jeder Zweite Tusk, während die PiS von weniger als 25 Prozent gewählt wurde. Am anderen Ende des Bildungsspektrums kehrte sich dieses Verhältnis nahezu um. Von den Polen mit dem niedrigsten Bildungsgrad konnte Tusk lediglich ein Viertel für sich gewinnen, Kaczyński rund 45 Prozent. Die Verteilung der Wähler nach Bildungsgrad folgt bei der PSL weitgehend dem PiS-Muster. Nur bei der Linksallianz ist das Ergebnis weniger eindeutig. Hier zeigt sich eine weitgehend gleichmäßige Unterstützung durch Wähler mit höherem und mittlerem Bildungsabschluss. Lediglich bei Polen mit beruflicher Bildung und den am schlechtesten Ausgebildeten fällt die Zustimmung mit rund elf beziehungsweise zehn Prozent (im Vergleich zu jeweils rund 14 Prozent) hier leicht zurück.

Eine ähnliche Verteilung ist beim Alter der Wähler zu beobachten - zumindest bei PO und PiS. Mit fast 54 Prozent war die Plattform bei den 18- bis 24-Jährigen am stärksten. Auch hier konnte die PiS weniger als 25 Prozent überzeugen. Dieses Übergewicht verteidigte die PO, bei gradueller Abschwächung bis ins Lager der 40- bis 59-Jährigen, wo beide Parteien jedoch mit 37,6 zu 34,6 Prozent fast gleichauf lagen. Erst bei den über 60-Jährigen kehrt sich das Verhältnis um; bei ihnen verbuchte die Partei der Kaczyńskis eine Unterstützungsrate von über 40 Prozent, während die Bürgerplattform deutlich unter 30 Prozent blieb. Sowohl die PSL als auch das Linksbündnis weisen hier jedoch eher Ähnlichkeiten zur PiS auf, das heißt, sie finden umso mehr Unterstützung, je älter die Wähler sind. Unterscheidet man die Wählerschaft nach Geschlecht, so fällt ein leichtes Übergewicht der Frauen unter den PO-Wählern auf; alle übrigen Parteien haben etwas mehr Unterstützung von Männern erhalten. ${ }^{26}$

25 Vgl. Maciej Kochanowski, Wielkie Miasta na PO (Die großen Städte für die PO), in: Gazeta Wyborcza vom 23. Oktober 2007 (http://wyborcza.pl/1,76842,4603631.html, Abruf am 25. Mai 2008).

26 Vgl. PBS DGA. 


\subsection{Wählermotive}

Betrachtet man diese Ergebnisse im Zusammenhang mit einigen zentralen sozialen Entwicklungen, so lassen sich wichtige Motive für den Sieg der Bürgerplattform und den damit verbundenen Regierungswechsel identifizieren. Zum ersten Mal durften Polen, die nach 1989 geboren sind, an die Urnen, und gerade bei Jung- und Erstwählern genoss die PO besonderen Rückhalt, während sie sich in den Kazcyński-Themen der Dekommunisierung und deren provinziell gefärbten Ressentiments gegen Fortschritt, kulturelle Vielfalt und das (west-)europäische Ausland kaum wiederfanden. Besonders deutlich wurde dies bei Studenten sowie bei Künstlern und Intellektuellen, die mit großer Mehrheit die Bürgerplattform unterstützten. Vor allem die im Ausland lebenden Polen, die die Vorteile der Öffnung nach außen direkt erfahren durften, konnten den antieuropäischen Tönen von Kaczyńskis Wahlkampf wenig abgewinnen. So stimmten von den in Schottland lebenden Polen rund 75 Prozent für die PO. ${ }^{27}$

Dabei spielte auch die Person des Spitzenkandidaten eine wichtige Rolle. So stand auf der einen Seite der weltoffene, jugendlich wirkende, fünfzigjährige Tusk mit seinem klaren Bekenntnis zu einem modernen, toleranten und weltoffenen Polen, auf der anderen Seite sein älterer Rivale Kaczyński, der das Land als von Feinden umzingelt betrachtete, überall Verräter witterte und von seinem Herausforderer im TV-Duell erfolgreich als hinterwäldlerischer Extremist dargestellt wurde, der selbst davor nicht zurückschreckt, politische Gegner mit der Waffe zu bedrohen. ${ }^{28}$ Das Aufeinandertreffen der beiden Spitzenkandidaten vor laufenden Kameras war insofern auch eine Begegnung zweier Modelle einer Gesellschaft, die in den vorangegangenen zwei Jahren von der Regierung in einer zuvor nicht gekannten Weise polarisiert worden war. Ungewollt mobilisierte Kaczyński mit diesem politischen Kurs die bis dahin weitgehend politisch inaktiven Teile dieser Gesellschaft, die immer stärker das Hineinwachsen eines notorisch misstrauischen Regierungsapparates in ihr persönliches Leben spürten.

Aufgrund des steigenden Bildungsniveaus könnte man darauf schließen, dass diese Wahlen eine Zäsur in der polnischen Nachwendegeschichte darstellen, durch die eine rund zwei Jahre dauernde Anomalie wieder korrigiert wurde und dass die Basis von PiS aufgrund der generellen sozioökonomischen Entwicklungstrends unweigerlich einem Erosionsprozess preisgegeben ist. Dem steht jedoch die wachsende Alterung der Gesellschaft gegenüber. Zudem lebt in Polen immerhin rund ein Drittel der Bevölkerung auf dem Land, und es sollte in diesem Zusammenhang auch nicht vergessen werden, dass PiS kein Wahlverlierer ist. Immerhin gewann die Partei im Vergleich zu den Wahlen von 2005 noch hinzu. Darüber hinaus besteht eine gewisse Nähe zwischen ihr und der Bürgerplattform. So hätte sich eine starke Strömung der PO lange Zeit durchaus eine Koalition mit der PiS vorstellen können, und auch die vielen personellen Wechselbewegungen zwischen beiden Gruppie-

27 Und dort, wo der direkte Kontakt zu den deutschen Nachbarn am intensivsten ist, stach auch die antideutsche Karte nicht, während in den von deutschen Minderheiten dominierten Gebieten (aber auch in Regionen mit einem hohen Anteil an Weißrussen) die nationale Identität in den Hintergrund trat und die politische Heimat weniger bei eigenen Vertretungen als bei der Bürgerplattform gesehen wurde. Vgl. Janusz A. Majcherek, a.a.O., S. 4.

$28 \mathrm{Vgl}$. Konrad Schuller, Tusks Wahlkampf mit Kaczynskis Pistole, in: Frankfurter Allgemeine Zeitung vom 15. Oktober 2007, S. 3. 
rungen sprechen für Übereinstimmungen. Hinzu kommt, dass auch die PSL klar auf den konservativen Koordinaten des polnischen Parteiensystems zu finden ist.

\section{Die Regierungsbildung}

In Bezug auf die präferierten Werte war die Bildung einer Regierung aus PO und PSL daher eine durchaus passende Option, denn um allein regieren zu können, fehlten Tusk und seiner Partei 22 Sitze. Eine Koalition mit der PiS verbat sich von selbst, während ein Zusammengehen mit der durch ihre Vergangenheit belasteten Linksallianz bestenfalls die zweite Wahl gewesen wäre. Zudem zeigten PO und PSL eine Reihe von Übereinstimmungen auch über die konservativen Werte hinaus. So strebten beide eine Verbesserung der Beziehungen zur Europäischen Union und Deutschland sowie zu Russland an. Problematisch erschien längere Zeit die Haltung zur Privatisierung und einer schnellen Euro-Einführung sowie die von Tusk angekündigte Einführung eines linearen Steuersatzes.

Nachdem die PO Tusk offiziell zum Kandidaten für das Amt des Premierministers gekürt hatte, kündigte er umgehend die Absicht an, ein Regierungsbündnis mit der PSL des Ex-Premiers Pawlak eingehen zu wollen. Am 5. November reichte Jarosław Kaczyński bei seinem Bruder den Rücktritt ein, der daraufhin die restlichen Mitglieder der Regierung traditionsgemäß ebenfalls zum Rücktritt aufforderte. Dann wurde innerhalb der durch die Verfassung (Art. 154) vorgegebenen Frist von vierzehn Tagen der Kandidat der stärksten Partei vom Präsidenten mit der Regierungsbildung beauftragt. Am 16. November 2007 wurden Tusk und der von ihm gebildete Ministerrat durch den Präsidenten ernannt. Tusk war nun offiziell polnischer Premier und hatte laut Gesetz genau zwei Wochen Zeit, um seine Regierung und deren Programm dem Sejm zu präsentieren und sich das Vertrauen aussprechen zu lassen. Am 23. November votierte die erforderliche Mehrheit des Sejm mit 238 gegen 204 Stimmen für das neue Kabinett.

Das Kabinett besteht zu einem großen Teil aus relativ unbekannten Namen. Einer der beiden Stellvertreter von Premier Tusk ist PSL-Chef Pawlak, der das Wirtschaftsressort übernommen hat. Außerdem entsandte die PSL den Landwirtschaftsminister Marek Sawicki und die Arbeits- und Sozialministerin Jolanta Fedak in die Regierung. Parteiunabhängige Kabinettsmitglieder sind der Finanzminister Jacek Rostowski, lange Zeit Professor an der London School of Economics, sowie Justizminister Zbigniew Cwiąkalski, Professor für Strafrecht an der Jagiellonen-Universität Krakau. Hinzu kamen Umweltminister Maciej Nowicki, die Ministerin für Regionalentwicklung Elżbieta Bieńkowska und die Ministerin für Nationale Erziehung Katarzyna Hall.

Zweiter Stellvertreter des Ministerpräsidenten wurde Innenminister Grzegorz Schetyna, der Generalsekretär der PO ist und als engster Vertrauter Tusks gilt. Weitere Minister der Bürgerplattform sind Bogdan Klich (Verteidigung), zwischen 1997 und 2001 Vizeverteidigungsminister unter der AWS-Regierung Jerzy Buzeks, Bogdan Zdrojewski (Kultur und Nationalerbe), ehemaliger Bürgermeister von Breslau, sowie Aleksander Grad (Schatzminister) und Ewa Kopacz (Gesundheit), zudem der Rechtsanwalt Cezary Garbarczyk (Infrastruktur) und Barbara Kudrycka (Wissenschaft und Hochschulwesen). Für Unruhe sorgte die Ernennung des Ex-Verteidigungsministers unter Kaczyński, Radosław Sikorski, zum Außenminister der neuen Regierung. Aufgrund des Zerwürfnisses von Sikorski mit den Zwillingsbrüdern lehnte der Präsident zunächst ab, diesen zu akzeptieren. Aus der Vorgabe der Verfassung, 
dem Präsidenten fielen auch Aufgaben im Bereich der Außenpolitik zu, leitete er die Auffassung ab, er könne auch bei der Besetzung des Außenministeriums mitentscheiden. Am Ende akzeptierte das Staatsoberhaupt zähneknirschend, wobei aus dem PiS-Umfeld verlautete, dass im Gegenzug Kaczyński keine neuen Botschafter ernennen und keine alten abberufen werde. $^{29}$

Neben der tagelangen Weigerung Lech Kaczyńskis, dem Wahlsieger zu gratulieren und der Ankündigung, unliebsame Gesetzesvorlagen notfalls zurückzuweisen, war dies ein weiteres deutliches Zeichen, dass die Kohabitation für die neue Regierung zu einer Bürde werden würde. Der Präsident kann fast jedes Gesetz an den Sejm zurückverweisen, der es dann nur mit einer Dreifünftel-Mehrheit doch noch verabschieden kann. Dieses letzte Mittel hat er bislang zwar nicht angewendet, dennoch knirscht es immer wieder im interinstitutionellen Gebälk. Als willige Helfer erweisen sich hier nicht zuletzt viele Funktionsträger der ehemaligen PiS-Regierung, die nun im Präsidialamt untergekommen sind, wie die ehemalige Außenministerin und jetzige Kanzleichefin im Präsidentenpalast Anna Fotyga.

Die EU-Charta der Grundrechte war das erste Thema, an dem Präsident und Regierung ihre Kräfte maßen. Am Ende behielt Kaczyński die Oberhand und erreichte, dass Polen dem britischen Beispiel des Opt-out folgte, so dass die Charta nun auch für Warschau nicht verbindlich ist. Im Gegenzug willigte er ein, die Ratifizierung des EU-Vertrages nicht zu blockieren. Es folgte die Rückberufung des Außenministers von einer wichtigen EU-Ministerratssitzung durch den Präsidenten, der, wie sich später herausstellte, nur gern etwas plaudern wollte. Auch bei der geplanten Anerkennung des Kosovos legte er Einspruch ein, da dies Georgien vor dem Hintergrund seiner Probleme mit Separatisten verprellen könne. Die Meinungen der Beobachter gehen darüber auseinander, ob der mangelnde Reformeifer auf inhaltliche Differenzen zwischen den Koalitionspartnern oder auf diese Zermürbungstaktik zurückzuführen ist. Fest steht, dass sich die Stimmen mehren, die fordern, die angekündigten Reformvorhaben endlich in die Tat umzusetzen. ${ }^{30}$ Sollte die Regierung Tusk dabei scheitern, erscheint es nicht unwahrscheinlich, dass ein neues Kapitel der polnischen Politik die Handschrift der Kaczyńskis tragen wird.

\section{Zusammenfassung: Wahlen 2007 eher eine oberflächliche Beruhigung}

Die Erleichterung über den polnischen Regierungswechsel im In- und Ausland mag berechtigt gewesen sein. Sie verkannte jedoch das Gewicht dieses Wechsels, das tatsächlich weniger gravierend ist, als der Erdrutschsieg der Bürgerplattform vermuten ließ. Zahlreiche Entwicklungen der Nachwendevergangenheit setzten sich bei den Wahlen 2007 fort:

29 Eine weitere sensible Personalie stellte die Ernennung Wtadisław Bartoszewskis zum Sonderbeauftragten für die polnisch-deutschen und die polnisch-israelischen Beziehungen dar, der die Kaczyńskis im Wahlkampf scharf angegriffen und der Lächerlichkeit preisgegeben hatte.

30 So erklärten in einer Umfrage vom Mai 2008 rund fünfzig Prozent der Befragten, dass die Regierung bisher ihre Erwartungen nicht erfüllt habe, rund zwei Drittel nennen hierbei insbesondere die dringend notwendige Reform des Gesundheitswesens (vgl. Tusk: Moi Rząd nie spełnia oczikewania, in: Gazeta Wyborcza Online vom 15. Mai 2008, http://www.dziennik.pl/polityka/article173945/Tusk_Moj_rzad_nie_spelnia_oczekiwan.html); vgl. auch Reinhold Vetter, Polens Reformern geht die Puste aus, in: Handelsblatt vom 11. Juni 2008 sowie Florian Kellermann, Polen manövriert sich in den Reformstau, in: Financial Times Deutschland vom 26. März 2008. 
(1) Die Alternation bleibt ein zentrales Charakteristikum polnischer Wahlen. Seit 1991 stand keine Regierung mehr als eine Wahlperiode durch.

(2) Wie praktisch alle Koalitionen vor ihr, so brach auch die von der PiS 2006 gebildete bald auseinander, so dass das Land vor den Neuwahlen von einer Minderheitsregierung geführt wurde.

(3) Wie bei den meisten Wahlen zuvor repräsentiert auch die neue Regierung unter dem in deutschen Medien meist als „Liberalen“ titulierten Tusk ideologisch heterogene, teilweise antagonistische Koalitionäre. So steht die Bürgerplattform als stärkste Kraft ökonomisch vor allem für eine Politik der Deregulierung, während der Juniorpartner, die aus einer ehemaligen sozialistischen Blockpartei hervorgegangene Bauernpartei PSL, klar interventionistisch orientiert ist. Vor dem Hintergrund des immens hohen Reformdrucks, der durch die sozialpolitische Untätigkeit ihrer konservativen Vorgänger noch erhöht wurde, verwundert es nicht, dass der Reformstau auch rund ein Jahr nach den Wahlen unvermindert ist. Dabei wirkt erschwerend die zweite Kohabitation in der polnischen Nachwende-Geschichte, diesmal mit dem weiterhin amtierenden Präsidenten Lech Kaczyński, der noch in der Wahlnacht klar machte, dass er sich weniger als neutrales Staatsoberhaupt denn als Erfüllungsgehilfe der politischen Ziele seiner Partei beziehungsweise seines Zwillingsbruders versteht. Er hat bereits mehrfach gedroht, Gesetzesvorhaben der neuen Regierung zu blockieren und damit den Reformeifer zusätzlich gebremst.

Hinzu kommt, dass durch die Wahlen von 2007 eine neuere Entwicklung des Parteiensystems revidiert wurde. Nachdem 2002 eine Reihe von populistischen und nationalistischen Gruppierungen in den Sejm katapultiert worden waren, deren zentrales Thema der drohende Ausverkauf der nationalen Souveränität durch den nahenden EU-Beitritt war, gelang es den Kaczyńskis, ihren lange gehegten (und bereits 2003 öffentlich angekündigten) Plan in die Tat umzusetzen, die beiden stärksten Gruppierungen dieses Lagers durch die Einbindung in eine Regierungskoalition zu entzaubern. Zunächst wurden die Samoobrona sowie die Liga der Polnischen Familien in eine „informelle“ Koalition aufgenommen, dann durch offizielle Regierungsmitgliedschaft „geadelt“ und schließlich im Zuge eines Kaczyńskitypischen Intrigenspiels erst bloßgestellt und danach aus der Regierung geworfen. Nach vernichtenden Ergebnissen schafften sie den Sprung über die Fünf-Prozent-Hürde und damit den Wiedereinzug in den Sejm nicht mehr. Dies heißt jedoch nicht, dass sich dadurch die politische Szene in Polen automatisch grundlegend verändert oder beruhigt hätte. Hier kommt eine weitere Konstante der polnischen Demokratie nach 1989 zum Tragen:

(4) Die große Instabilität der Parteien, die sich in einer hohen Fluktuation ihrer führenden Persönlichkeiten, teilweise sogar über die politischen Lagergrenzen hinweg, äußert. So trat der ehemalige polnische Ministerpräsident und langjährige Vorsitzende der ex-kommunistischen Sozialdemokratie, Leszek Miller, der von Leppers SO wegen seiner Unterschrift unter den EU-Beitrittsvertrag noch als „Vaterlandsverräter“ beschimpft worden war, bei den Wahlen 2007 von der Liste der Samoobrona an. Und der von Kaczyński entlassene PiSVerteidigungsminister Radostaw Sikorski ist nun unter seinem ehemaligen Konkurrenten Tusk zum Außenminister avanciert. Es ist unverkennbar, dass die Parteien weiterhin eher Vehikel der politischen Ambitionen ihrer Führungsfiguren als Repräsentanten des Wählerwillens beziehungsweise bestimmter gesellschaftlicher Partikularinteressen darstellen.

Daher ist zwar zu erwarten, dass die politischen Auseinandersetzungen nach dem Ausscheiden der populistischen Scharfmacher von SO und LPR aus dem Sejm in der laufenden Wahlperiode etwas weniger turbulent ausfallen werden. Persönliche Ambitionen und poli- 
tisches Taktieren führten in Polen aber immer wieder zum Zerbrechen der fragilen Koalitionen mit personell wie programmatisch oftmals kaum zu integrierenden Parteien. Die gegenwärtige Koalition muss hier keine Ausnahme bilden. Wenn sie bisher auch nicht an Querelen zwischen den Partnern gescheitert ist, so erscheint es durchaus möglich, dass sie durch eine Unfähigkeit zu Reformen ebenfalls Gefahr läuft, das Schicksal der vergangenen Regierungsbündnisse zu teilen. Die Kohabitation mit dem stark parteilich agierenden Präsidenten könnte hierzu weiter beitragen.

Darüber hinaus ist davon auszugehen, dass die nächsten Wahlen keine dem Jahr 2007 vergleichbare Mobilisierung erleben werden. Insbesondere bei einem Scheitern der Regierung wird aller Voraussicht nach die Enttäuschung zu großer Wahlenthaltung führen, die traditionell eher der PiS nutzt, die über eine treue und wahlwillige Stammwählerschaft verfügt. Nicht umsonst riefen führende PiS-Politiker kurz vor dem Wahltermin im vergangenen Oktober beinahe unverhohlen dazu auf, den Wahlen fernzubleiben. Eine dauerhafte Veränderung wird jedoch wahrscheinlich das Verschwinden von Samoobrona und der Liga der Polnischen Familien von der politischen Bühne sein. Eventuell können Teile dieser Gruppierungen organisatorisch in der PiS aufgehen. Ihr Kernthema, der Kampf des traditionellen, ländlichen Polen gegen den modernen, vom Ausland dominierten städtischen Teil des Landes, haben die Kaczyńskis gegenwärtig fest besetzt. ${ }^{31}$ Dieses Thema wird im Verlauf der weiteren sozioökonomischen Transformation des Landes noch längere Zeit von erheblicher Bedeutung bleiben. Und so lange wird auch PiS ein bestimmender Akteur auf der politischen Bühne in Warschau sein.

In der Gesamtschau ist der Regierungswechsel in Polen insofern eher als oberflächliche Beruhigung denn als Ankerpunkt einer mittel- oder gar langfristigen Stabilisierung des politischen Gefüges zu sehen. Und er war auch nicht ein besonderer Ausweis demokratischer Reife der polnischen Bevölkerung angesichts bedenklicher Machtpraktiken der alten Führung, sondern eher das übliche Zeichen des Verdrusses über eine weitere abgewirtschaftete Regierung.

31 Den größten Teil ihrer Unterstützung zogen sie zudem aus der Angst vor den möglichen negativen Folgen des EU-Beitritts. Letztlich war zu erwarten, dass bei einem dauerhaften Ausbleiben der ausgemalten Schreckensszenarien auch das Interesse an seinen Erfindern und Pflegern über kurz oder lang abebben würde.

\title{
Die Transformation des russischen Parteiensystems: Regimestabilisierung durch personalisierte Institutionalisierung
}

\author{
Petra Stykow
}

Die Bilanz der achtjährigen Präsidentschaft Vladimir Putins (Januar 2000 bis Mai 2008) ist zwiespältig: Einerseits wurde in diesem Zeitraum mit einem anhaltenden Wirtschaftswachstum von jährlich über sechs Prozent, gewachsener politischer und sozialer Stabilität sowie der Rückkehr Russlands in die globale Arena eine einzigartige Erfolgsgeschichte geschrieben. 